\title{
S13 File
}

Mutation rate, diversity and eveness across the polyprotein

\# Snps per 100bp Shannon diversity index Shannon equitability

Early Aegypti $\begin{array}{lll}0.397 & 1.098 & 0.187\end{array}$

Early Albopictus

$\begin{array}{lll}0.501 & 1.22 & 0.2\end{array}$

Early Human

0.438

1.314

0.22

Late Aegypti

0.536

1.071

0.173

Late Albopictus

0.406

1.152

0.195

Late Human

0.566

1.285

0.206

S13 File. Mutation rate, diversity and eveness across the polyprotein. The number of mutations in a $100 \mathrm{bp}$ window were calculated across the genome. Diversity and evenness of the nucleotide type change $(\mathrm{A} \leftrightarrow \mathrm{G}, \mathrm{C} \leftrightarrow \mathrm{T}, \mathrm{A} \leftrightarrow \mathrm{C}, \mathrm{G} \leftrightarrow \mathrm{T}, \mathrm{G} \leftrightarrow \mathrm{C}, \mathrm{A} \leftrightarrow$ $\mathrm{T})$ were calculated using the Shannon diversity index and Shannon equitability measurement respectively 